http://dx.doi.org/10.35381/r.k.v5i5.1037

\title{
Percepciones docentes frente a la Educación Inclusiva Superior
}

Teachers' perceptions against Higher Inclusive Education

\author{
Catalina Patricia Muñoz-León \\ catalina.munoz.43@est.ucacue.edu.ec \\ Universidad Católica de Cuenca, Azogues \\ Ecuador \\ https://orcid.org/0000-0002-6900-3061 \\ Darwin Gabriel García-Herrera \\ dggarciah@ucacue.edu.ec \\ Universidad Católica de Cuenca, Azogues \\ Ecuador \\ https://orcid.org/0000-0001-6813-8100 \\ María Isabel Álvarez-Lozano \\ mialvarezl@ucacue.edu.ec \\ Universidad Católica de Cuenca, Biblián \\ Ecuador \\ https://orcid.org/0000-0001-8029-1933 \\ Juan Carlos Erazo-Álvarez \\ jcerazo@ucacue.edu.ec \\ Universidad Católica de Cuenca, Cuenca \\ Ecuador \\ https://orcid.org/0000-0001-6480-2270
}

Recibido: 15 de septiembre de 2020

Revisado: 19 de octubre de 2020

Aprobado: 15 de noviembre de 2020

Publicado: 01 de diciembre de 2020 


\title{
RESUMEN
}

Se tiene por objetivo analizar las percepciones docentes frente a la Educación Inclusiva Superior del Ecuador. Metodológicamente fue de tipo descriptiva no experimental de cohorte transversal ya que se realizó en un solo momento. La población estuvo conformada por 34 docentes de la Universidad Católica de Cuenca (UCACUE). Se determina que los docentes de la Unidad Académica de Educación de la Universidad Católica de Cuenca [UCACUE], poseen percepciones positivas frente a la inclusión debido a la cantidad de alumnado con varias discapacidades que acoge la universidad, en su totalidad 154. Adicionalmente estos resultados se logran gracias a la difusión de políticas inclusivas internas y el trabajo conjunto que realizan los departamentos de Bienestar Estudiantil y la Unidad de Diagnóstico, Investigación Psicopedagógica y Apoyo a la Inclusión [UDIPSAI] aportando de manera directa a la disminución de barreras y retos que enfrentan los docentes dentro de sus aulas.

Descriptores: Enseñanza científica superior; política educacional; indicadores educativos. (Palabras tomadas del Tesauro UNESCO).

\begin{abstract}
The objective is to analyze teachers' perceptions regarding Higher Inclusive Education in Ecuador. Methodologically, it was of a descriptive non-experimental cross-sectional cohort type, since it was carried out in a single moment. The population was made up of 34 teachers from the Universidad Católica de Cuenca (UCACUE). It is determined that the teachers of the Academic Unit of Education of the Catholic University of Cuenca [UCACUE], have positive perceptions of inclusion due to the number of students with various disabilities that the university welcomes, in its entirety 154. Additionally, these results They are achieved thanks to the dissemination of internal inclusive policies and the joint work carried out by the Student Welfare departments and the Unit for Diagnosis, Psychopedagogical Research and Support for Inclusion [UDIPSAI], contributing directly to the reduction of barriers and challenges they face teachers inside their classrooms.
\end{abstract}

Descriptors: Higher science education; educational policy; educational indicators. (Words taken from the UNESCO Thesaurus). 


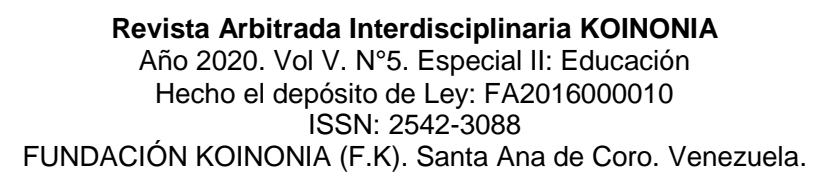

Catalina Patricia Muñoz-León; Darwin Gabriel García-Herrera; María Isabel Álvarez-Lozano Juan Carlos Erazo-Álvarez

\section{INTRODUCCIÓN}

La educación es sin duda un tema que provoca constantes observaciones y análisis en los diferentes contextos alrededor del mundo. Los paises abordan la educacion dentro de sus politicas de estado apegandose a las normas internacionales pero tambien a sus realidades individuales. Sin embargo, independientemente del nivel socioeconómico de un país, el acceso a la educación implica, movimientos, interés público y privado, normas sociales y políticas, pero sobretodo convenios entre países, entidades y organizaciones que buscan de manera continua una mejora a la educación en todos sus niveles.

Todo esto conlleva a un desafío que desencadena en un compromiso social enfocado desde la accesibilidad a la educación, hacia la calidad de la misma. Es así como la Organización de las Naciones Unidas para la Educación, la Ciencia y la Cultura [UNESCO] en su Agenda 2030 establece mediante el 4to Objetivo de Desarrollo Sostenible [ODS] que la educación debe ser inclusiva, debe ser para todos, priorizando dos características indispensables: equidad e igualdad, mismas que considera como las vías directas para alcanzar la calidad educativa. Enfatiza también sobre la necesidad de contar con maestros debidamente calificados en términos educativos y ciudadanos.

Por otro lado, también se propone un nuevo enfoque, y evaluación a las instituciones educativas a manera de promover y velar por el cumplimiento de normas en torno a la inclusión en la educación. Una capacitación del docente de manera continua y un cambio en la concepción de calidad educativa son las propuestas claves para el cumplimiento de una educación igualitaria y universal (Organización de las Naciones Unidas para la Educación, la Ciencia y la Cultura, UNESCO, 2015).

La UNESCO es quizá, la entidad que más esfuerzos a realizado por esparcir la necesidad de la inclusión en la educación calificándola como estrategia clave para fortalecer el sistema educativo y el desarrollo de regiones para con ello transformar vidas. Sin embargo, de acuerdo con los estándares internacionales en cuanto a políticas inclusivas es necesario recalcar la convención sobre los derechos de las personas con 


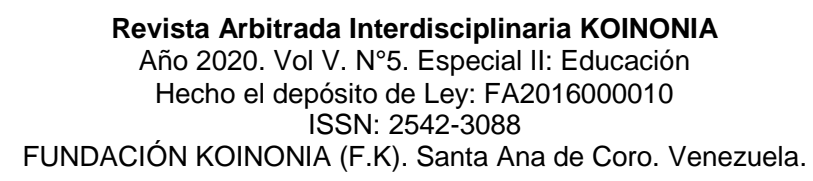

Catalina Patricia Muñoz-León; Darwin Gabriel García-Herrera; María Isabel Álvarez-Lozano Juan Carlos Erazo-Álvarez

discapacidad, pasando de abordarse la formación de este grupo de personas de manera separada a una conjunta dentro de la educación convencional.

Pero el adoptar políticas integradoras requieren también un cambio de ideología y trabajo colaborativo entre educadores, especialistas y organismos de control. En tal sentido la (UNESCO, 2019), reconoce a la Educación Inclusiva como la eliminación de barreras que impiden el acceder a la educación en igualdad de condiciones y oportunidades que el resto de los alumnos. Se trata primordialmente de brindar el acceso y oportunidad de cambio a grupos humanos que comúnmente han estado expuestos a la discriminación. Uno de los grupos humanos que han enfrentado la vulnerabilidad en su educación son las personas con discapacidad.

Enfrentar barreras y limitaciones legales no es el único desafío, también deben lidiar con barreras y obstáculos actitudinales. Situaciones de miedo, burlas o rechazo son algunos de los tratos recibidos. Varias son las causas; entre ellas: el desconocimiento, la desinformación o simplemente una falta de empatía. Si bien el crecimiento de las sociedades y su población conlleva también a un incremento en la desigualdad social, es indispensable recalcar en el reconocimiento de las minorías como parte de una sociedad democrática, justa, no discriminadora y por lo tanto inclusiva.

Así por ejemplo el Instituto Internacional para la Educación Superior en América Latina y el Caribe [IESALC] en su informe de gestión 2011-2018 menciona que una de sus estrategias para mejorar la Educación Superior [ES] es la producción, articulación y vinculación de proyectos educativos y de investigación que permitan la internacionalización de la práctica educativa (UNESCO-IESALC, 2018). Adicionalmente la Conferencia Regional de Educación Superior [CRES] en su informe 2018 enfatiza en la formación del docente frente a una sociedad que demanda oportunidad de igual acceso a una calidad educativa. En sus líneas propone una continua y permanente capacitación del nuevo docente en torno a las necesidades sociales en las que se desenvuelve y refiere: 
Revista Arbitrada Interdisciplinaria KOINONIA

Año 2020. Vol V. N5. Especial II: Educación

Hecho el depósito de Ley: FA2016000010

ISSN: 2542-3088

FUNDACIÓN KOINONIA (F.K). Santa Ana de Coro. Venezuela.

Catalina Patricia Muñoz-León; Darwin Gabriel García-Herrera; María Isabel Álvarez-Lozano Juan Carlos Erazo-Álvarez

Por tanto, su formación inicial y continua debe responder a un nuevo paradigma docente, respaldado por modelos formativos que lo habiliten como actor clave del cambio educativo que requiere ALC en una sociedad globalizada, lo que es viable cuando se integran la dimensión internacional y la visión universal en la formación de los profesores" (CRES-IESALC, 2018.p.60).

Es así como, el termino inclusión comienza a tomar fuerza basándose en una flexibilidad curricular y sistemática. Un nuevo enfoque donde la diferencia y la diversidad son ventajas del sistema educativo que convierten a la práctica docente en un desafío hacia el progreso individual y social (Organziación de las Naciones Unidas (ONU), 2008). Se puede afirmar que el marco de la educación exige que aquellas características de equidad e igualdad se conviertan en principios educativos. El principio de Igualdad en dignidad hacia todo educando y principio de equidad en cuanto a oportunidades de aprender y participar. Solo la evidencia del disfrute pleno de un convivir dentro de la etapa estudiantil será el indicador de un trabajo en el cumplimiento del cuarto ODS y una educación para todos.

En el ámbito nacional la (Asamblea Nacional, 2008) en su art. 27 garantiza el derecho a la educación y menciona que "será participativa, obligatoria, intercultural, democrática, incluyente y diversa, de calidad y calidez" (p.8). Si bien la ley esta establecida, el cumplimiento de la misma se torna un tanto dificil debido al desconocimiento de las pautas y acciones concretas. La capacitacion y consiciencia social sobre inclusión es basicamente un cambio de pensamiento, percepciones y acciones. Es asi tambien como a partir de esta ley, la Secretaría Nacional de Planificación y Desarrollo [SENPLADES] centra sus esfuerzos en tres ejes que garantizan una mejora de vida social que parte de una educación inclusiva sobre todo en los niveles bachillerato y superior pues, son estos el escalon hacia la vida productiva y laboral de futuros profesionales que aportan al desarrollo de todo un pais (Secretaría Nacional de Planificación y Desarrollo - Senplades, 2017). 


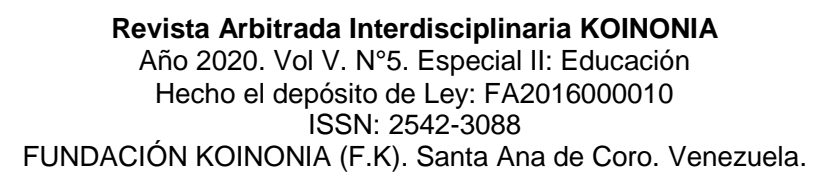

Catalina Patricia Muñoz-León; Darwin Gabriel García-Herrera; María Isabel Álvarez-Lozano Juan Carlos Erazo-Álvarez

Sin embargo los desafíos ante una educación inclusiva de calidad están encadenados. De nada sirve la ley si no se la ejecuta. Si bien las instituciones de ES declaran la inclusión como parte de sus políticas, varios estudios realizados hablan de una mera integración de estudiantes, más no de una inclusión estructurada y planificada que se considere una guía para las partes que integran el proceso educativo. Como mencionan (Echeita \& Ainscow, 2011), los maestros son la clave en el proceso de enseñanza aprendizaje pues son los encargados de socializar y ejercer, de manera directa en las aulas, las normativas que proponen las autoridades de educación.

Se tiene por objetivo analizar las percepciones docentes frente a la Educación Inclusiva Superior del Ecuador.

\section{Referencial teórico}

El crecimiento de las sociedades y su población conlleva también un incremento en la desigualdad social. Es así como la (UNESCO, 2015), en su Declaración de Incheón, junto con otras organizaciones aprueban la -Agenda para la Educación 2030- misma que otorga un valor importante a la educación, y en su cuarto Objetivo de Desarrollo Sostenible [ODS] establece velar para que los docentes se empoderen y sean debidamente contratados gracias a su buena formación que garantice su profesionalismo. Que se sientan motivado y apoyados por los sistemas de educación que les facilite los recursos suficientes y eficaces. Del mismo modo la Conferencia Regional de Educación Superior [CRES] enfatiza claramente que uno de los principios es la eliminación de cualquier tipo de discriminación o segregación social (CRES, 2018).

La exclusión de las personas con discapacidad, terminología que según la Organización Mundial de la Salud [OMS] refiere a todo tipo de deficiencia, que limita las actividades y restringe la participación del individuo que la afronta ha sido durante mucho tiempo una realidad que sobrepasa el avance y desarrollo de una región. La discapacidad en un acontecimiento que guarda estrecha relación entre las condiciones del organismo humano y las condiciones del contexto social al que pertenece. Es decir, factores 


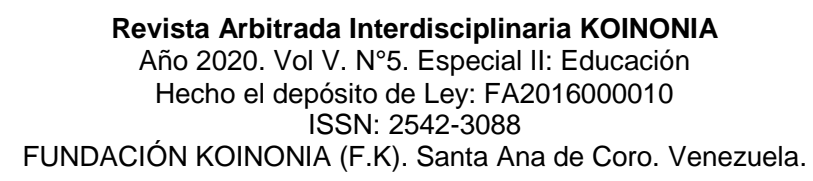

Catalina Patricia Muñoz-León; Darwin Gabriel García-Herrera; María Isabel Álvarez-Lozano Juan Carlos Erazo-Álvarez

personales deficiencias y contextuales -barreras- se interrelacionan, así la conducta de unos infiere en el accionar de otros (OMS, 2011), se requiere empatía y educación conjunta para que ninguna de las partes obstaculice la cultura inclusiva que se pretende conseguir

A pesar de ello, existen casos de alumnos con discapacidad que aun con sus limitaciones pueden tener una inserción educativa y laboral siempre que el estado y la sociedad brinden las herramientas y condiciones necesarias para su realización. La Educación es una de las herramientas clave para el desarrollo humano y social, pero más allá de la generación de leyes, acuerdos internacionales y trabajo interinstitucional, hay que realizar que son los docentes los directamente implicados en perseguir el cumplimiento de la ley establecida. Una educación que satisfaga los intereses y necesidades de sus alumnos. La eliminación de barreas y limitaciones deben iniciar en las aulas y el trato mismo del docente. Es por ello que la tendencia de la enseñanza es otorgar un contenido educativo que impulse y asegure el bien estar y buen vivir de las personas. Que aliente a la superación personal y profesional, a un trabajo en comunidad con sentido de pertenencia a una comunidad.

Es así como el porcentaje de la demanda de matrículas en formación de tercer nivel ha tenido un buen incremento. En América Latina y el Caribe [ALC] según el Instituto de Estadísticas de la UNESCO [UIS], indica que en el año 2000 el porcentaje fue de $22.65 \%$ mientras que en el 2015 alcanzó el $46.52 \%$ de estudiantes que iniciaban su preparación profesional, evidenciando que el $11.7 \%$ del total de matrículas en ES a nivel mundial correspondía al contexto de ALC. (UIS, 2020). La capacitación constante y actualizada de docentes es la clave al mismo tiempo que la conciencia de un espíritu innovador, investigador con ansias de educar y educarse para que la transformación educativa sea real para todas las partes que la integran.

Por tal motivo los docentes deben ser los primeros en estar debidamente capacitados y preparados de modo que lo que se enseña sea de calidad y utilidad en un mundo cada vez más globalizado y exigente. Esta conciencia de preparación y estudio es igualmente 


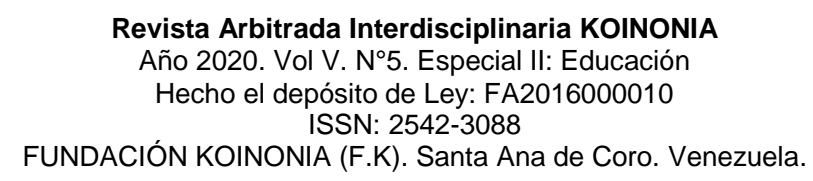

Catalina Patricia Muñoz-León; Darwin Gabriel García-Herrera; María Isabel Álvarez-Lozano Juan Carlos Erazo-Álvarez

reconocida por los jóvenes estudiantes que se esfuerzan por conseguir entrar a la universidad y contar con un título académico que avale su preparación.

Para (Booth \& Ainscow, 2002) el término inclusión engloba una serie de procesos enfocados a eliminar las barreras que entorpecen o anulan la participación social y el aprendizaje. Es decir barreras que pueden ser intra o interpersonales como por ejemplo una actitud desfavorable por parte de aquella gran comunidad educativa conformada por: el estado, la familia y la sociedad. Es así como el Index for Inclusion propuesto por estos dos autores servirá para analizar la inclusión en el contexto ES a través de sus tres dimensiones inclusivas: política, práctica y cultura.

Del mismo modo (Echeita \& Ainscow, 2011), mencionan que la inclusión educativa requiere por un lado, un esfuerzo de trabajo conjunto necesario entre organizaciones, y por otro, el despertar de la conciencia social para hacer frente a las barreras que limitan la práctica educativa inclusiva. En la actualidad es justo mencionar que se percibe una reflexión social respeto a los derechos y bienestar común, sin embargo el trabajo debe continuar de manera organizada, y bien articulada. El proposito no se centra en el cumplimiento de un ODS sino en el cumplimiento universal de un derecho humano como la educacion ligado a normas de calidad que fomenten el progreso y desarrollo peresonal y social.

En Malasia por ejemplo, el plan "No dejar a a nadie atrás" ["No one behind"] acoge la inclusion como decreto de gobierno lo que implica pasar de una investigacion disciplinar a una investigación pedagógica. Reconoce además que la inclusión es el mecanismo para una equidad en la educación y de habilidades basados en la justicia y bienestar de sus estudiantes. A pesar de ello, los indices de estudiantes matriculados disminuyeron desde el 2016 al 2018, básicamente debido aciertos factores como: la persistencia de una falta de preparación docente, definiciones inconsistentes de discapacidad y sus tipos, desconocimiento en el trato a personas con discapacidad (Rafy-Syed-Jaafar, et al., 2020). De nada sirve tener el amparo de reglamentos internacionales si lo que falta es la voluntad en si de las personas de trabajar hacia un cambio. 


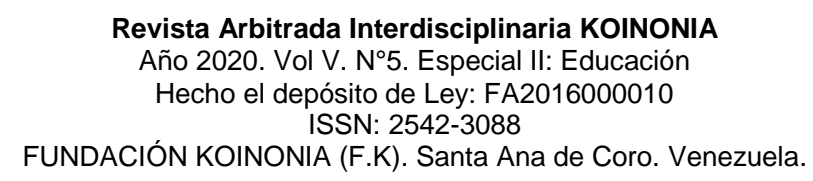

Catalina Patricia Muñoz-León; Darwin Gabriel García-Herrera; María Isabel Álvarez-Lozano Juan Carlos Erazo-Álvarez

Varios estudios han sido realizados en torno a la inclusión en la educación de tercer nivel. Así, se tiene dos investigaciones en España; por un lado, está la de (Moriña \& Melero, 2016), que analizan los factores de soporte para el éxito académico del estudiante con discapacidad, identificando la importancia de lo que denominan redes de apoyo- a nivel: familiar, social y académico. Por otro lado, está (Cotán, 2017), quien identifica las barreras y ayudas de una Universidad de Sevilla, a los estudiantes con discapacidad, demostrando que, si bien la institución había realizado modificaciones de acceso físico, aún faltaba adecuaciones como: softwares de lectura y programas de voz para personas con deficiencia visual, entre otros.

Entre las principales ayudas estaban el aparcamiento cercano a sus aulas, tutoría docente, designación de un -alumno/a colaborador- y entrega de temas con anterioridad por parte de los maestros. Aquello evidencia entonces que el estudiante con discapacidad necesita no solo de una aceptación familiar y social sino de un proceso de apoyo académico digno, que garantice una calidad educativa y que las distintas estrategias que adopte la institución guíen, acompañen y motiven su preparación profesional.

Una investigación en torno a la inclusión en Noruega, país en donde los avances tecnológicos y educativos son propios del primer mundo, refleja que los alumnos con discapacidades invisibles como TDHA, Dislexia, Ansiedad, Depresión, entre otros, requieren adaptaciones curriculares, pero también experticia por parte del docente.

Una manera de sensibilizar a los maestros ha sido el compromiso de realizar prácticas inclusivas en todas las instancias, sin embargo, esto desembocó en un estrés abrumador por parte de los docentes que no sabían cómo mantener los estándares académicos y al mismo tiempo realizar adaptaciones a los alumnos de inclusión (Svendby, 2020). El desconocimiento es lo que produce desconcierto y miedo. Saber que en algún momento todo docente enfrentará la inclusión en sus aulas, es algo que no se lo piensa al momento de escoger su carreara o su trabajo. Pensar en docencia es pensar en un trabajo de horas, de medio tiempo, y si es en los niveles iniciales, es pensar en juegos y cuidados. Claro está que, en torno a la educación inclusiva, además de existir una falta de cohesión 


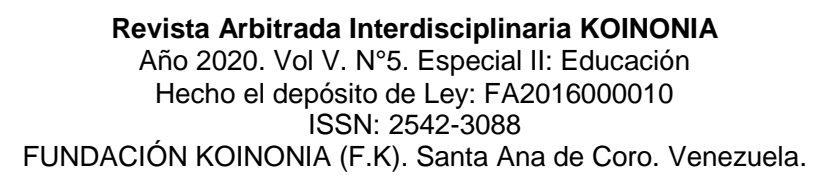

Catalina Patricia Muñoz-León; Darwin Gabriel García-Herrera; María Isabel Álvarez-Lozano Juan Carlos Erazo-Álvarez

entre la ley y la práctica, también existe un vacío, que es el ¿Cómo lo hago?, para esto es indispensable entregar herramientas y recursos que faciliten el mecanismo tanto de enseñar como de aprender.

Dentro del ámbito Latinoamericano se encuentran los estudios realizados por (GrossMartínez \& Stiller-González, 2014), en la Universidad de Costa Rica donde mediante talleres de capacitación docente en temas de discapacidad e inclusión, se comprueba las necesidades educativas institucionales de accesibilidad y actualización en la formación del docente. Esto es indispensable para asegurar que la educación inclusiva superior sea una realidad y pueda transformar positivamente el futuro de los educandos, más aún aquellos que; estando en situaciones vulnerables; tendrán que enfrentar un mundo laboral tan diverso como lo son sus miedos, capacidades y limitaciones.

En otro país de la región (Rojas-Rojas, et al., 2018), describen los intereses educativos de estudiantes con discapacidades visuales, auditivas, de habla y motora demostrando que para brindar una mejor calidad educativa requiere de adecuaciones a nivel institucional lo que llevó a la universidad a la implementación de un software que permita adecuar los contenidos pedagógicos a la traducción digital de mensajes en: lenguaje de señas, alfabeto braille y audiolibros y videos con sensores táctil y gestual. Este punto se puede destacar observando que la integración de personas con discapacidad dentro de la ES demanda una responsabilidad institucional que se acomode a las diversas necesidades y potencialidades de sus estudiantes.

Un siguiente aporte es el de (Vilchis-Romero \& Arriaga-Ornelas, 2018), en México, con un Estudio de Caso de un joven universitario con limitaciones visuales, mencionando que la principal barrera que ha tenido que superar es la de carácter social resultado de concepciones y actitudes desfavorables ante la discapacidad. Sin embargo, su permanencia en la institución ha sido gracias al soporte de la familia y persistencia propia. $\mathrm{Si}$ bien lo que se pretende es conocer las percepciones de docentes frente a la discapacidad en el aula, cabe entender que en el proceso de educación intervienen todos los actores, incluida la sociedad. Si la Educación Inclusiva tiene como objetivo una 


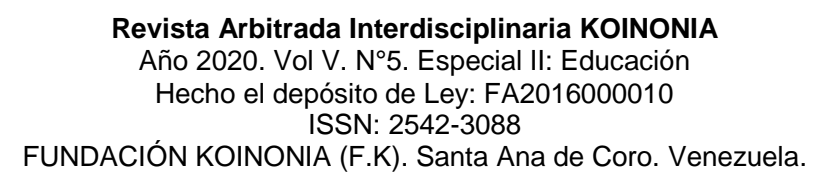

Catalina Patricia Muñoz-León; Darwin Gabriel García-Herrera; María Isabel Álvarez-Lozano Juan Carlos Erazo-Álvarez

Educación Para Todos [EPT] entonces es preciso concebir a la diversidad como una riqueza y no como un problema.

En Ecuador, la inclusión si bien está establecida en los reglamentos gubernamentales, aun, requiere ahondar esfuerzos por atender a la diversidad estudiantil y facilitar el acceso, permanencia y culminación. Es un derecho que requiere control, seguimiento y trabajo. En el área de ES lo demuestra el estudio que realiza (Ocampo, 2018), en la Universidad Católica de Santiago de Guayaquil, partiendo que desde el 2013 al 2017 el número de estudiantes con discapacidad matriculados fue en aumento, pero que aún existían facultades y carreras que estaban más preparadas que otras para recibir a estudiantes con discapacidad, y que el escaso archivo de información no permitió dar resultados exactos sobre estudiantes que egresaron, desertaron o tuvieron dificultades en ser promovidos de año.

Todo esto podría indicar la necesidad de un seguimiento, registro y actualización de datos que permitan conocer las razones de la inasistencia o deserción del estudiante con discapacidad. El objetivo principal de estas tareas es motivar y velar que los alumnos incluidos culminen sus estudios necesarios para una inserción laboral.

En cuanto al rol docente al contar con un estudiante de inclusión, se cuenta con la investigación de (Vélez-Calvo, et al., 2016), quienes comparan la formación del maestro en España y de Ecuador. Para ello se observó los planes de estudio de universidades en estos dos países arrojando una similitud en torno a la escasa formación en educación inclusiva un 59,44\% en España frente al 36,5\% en Ecuador con carreras con al menos una asignatura en torno a la discapacidad. Si lo que se quiere es generar un cambio a partir de la educación entonces los maestros como coprotagonistas del proceso de aprendizaje, deberán promover su ejercicio hacia una cultura inclusiva, con igualdad de oportunidades y participación. En la actualidad y sin importar la modalidad de estudio (presencial, virtual, semipresencial,) es importante que el alumno perciba un recibimiento, apoyo y acompañamiento por parte del maestro. 


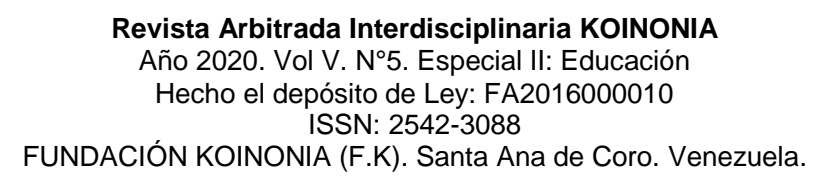

Catalina Patricia Muñoz-León; Darwin Gabriel García-Herrera; María Isabel Álvarez-Lozano Juan Carlos Erazo-Álvarez

Finalmente, el aporte más actual que realizan (Clavijo-Castillo \& Bautista-Cerro, 2020), explica los retos de la EIS en el Ecuador. Mencionan que, si bien los avances internacionales en torno a la inclusión educativa han provocado un adelanto en las leyes y normas nacionales, las políticas internas de los establecimientos ecuatorianos; tanto públicos como privados; aun reflejan un trabajo por hacer. La inclusión educativa no está enfocada únicamente a la discapacidad, se trata de un pensar, sentir y actuar en favor de crear una sociedad enriquecida con la consciencia de diversidad y el respeto mutuo. El ingreso a las universidades no es tarea fácil ni inmediata de conseguir para los estudiantes. En muchos casos, si no en todos, deben rendir un examen de admisión que mide tanto su capacidad académica como su habilidad en la resolución de conflictos. Ocurre en contextos internacionales y locales.

Si la Educación es un derecho y, la Inclusión es el nuevo enfoque educativo entonces, los docentes tienen un papel importante dentro de este proceso de transformación. Es por esto por lo que se sugiere la formación docente hacia estudiantes con discapacidad y/o Necesidades Educativas Especiales (NEE) en la misma medida que en educación general. No se da lo que no se tiene, por lo tanto, si el docente es el encargado de transmitir conocimiento y preparar al estudiante para la vida, deberá él también estar empoderado de conocimiento suficiente que permita este transmitir de calidad.

Dentro de los entes regulatorios de este tópico social se cuenta desde el año 2010 con la Ley Orgánica de Educación Superior [LOES] en la cual se establece la garantía de las condiciones necesarias de acceso para que las personas con discapacidad no encuentren limitación en el desarrollo de sus actividades y habilidades académicas. El objetivo principal de la educación inclusiva en todos sus niveles se cimienta en el deseo de dar origen a la superación del ser humano. No se trata de un tema de cumplimiento de leyes, se trata de voluntad, de empatía, de resiliencia social.

Referente a ello la (LOES, 2010), define a la ES como un bien común, enfocado a brindar igualdad de acceso y oportunidad de formación profesional. Las universidades se 


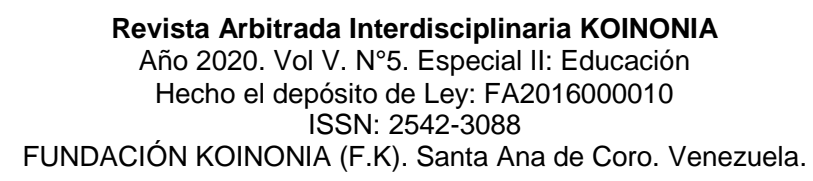

Catalina Patricia Muñoz-León; Darwin Gabriel García-Herrera; María Isabel Álvarez-Lozano Juan Carlos Erazo-Álvarez

encuentran en la obligación de velar por este cumplimiento. Más aún cuando de ellas depende la calidad de profesional que brinde a la sociedad.

Dentro de la educación el conocimiento es de doble vía, aprenden juntos tanto alumnos como maestros. Una especie de trueque en la que las dos partes quedan satisfechos. Establece la búsqueda de la calidad educativa desde una auto reflexión hasta una construcción colectiva y equilibrio entre la formación docente y la sociedad. Una inclusión pertinente y democrática apegada a la equidad, diversidad y autonomía. Es importante establecer que, si bien las leyes deben velar por la educación del alumno, también deberán hacerlo por la del maestro. Un ganar, ganar del proceso enseñanza aprendizaje, pues si es de doble vía también es de doble efecto.

Las acciones tomadas por el gobierno ecuatoriano miran hacia una inclusión de calidad para una vida digna y plena de todos sus ciudadanos, es por ello que, se requiere instar a las distintas organizaciones que no solo la promuevan, sino que la asuman con seriedad y compromiso. La formación del futuro maestro con la visión y misión de ejercer una educación inclusiva deberá darse desde sus inicios en las aulas universitarias procurando mejorar la vida de sus alumnos como las suyas propias.

Cabe destacar que dentro de las políticas ecuatorianas el Ministerio de Educación, ha creado conciencia de este fenómeno inclusivo y es por esto que el propósito de educar pasa de, una perspectiva integradora basada en un currículo individualizado, homogéneo, con principio de igualdad, a una perspectiva inclusiva basado en un currículo universal, heterogéneo, con principio de equidad (MINEDUC, 2011). En otras palabras, pasa de exigir la adaptación del estudiante al sistema educativo a brindar una flexibilidad de dicho sistema que asegure el aprendizaje de todos sus alumnos concibiendo a la diversidad como una riqueza socioeducativa.

Concretamente en lo que refiere a la educación de personas con discapacidad el Consejo Nacional para la Igualdad de Discapacidades [CONADIS] menciona su participación en la capacitación a 250 docentes en educación inclusiva, quienes a su vez replicaron esta preparación a colegas, llegando a 5000 docentes capacitados en este ámbito. Del mismo 


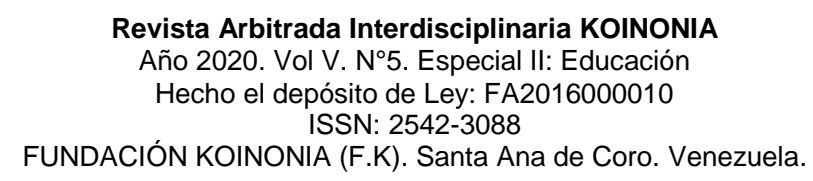

Catalina Patricia Muñoz-León; Darwin Gabriel García-Herrera; María Isabel Álvarez-Lozano Juan Carlos Erazo-Álvarez

modo la institución informa haber realizado adaptaciones al examen Ser bachiller para que pueda ser ejecutado por personas con discapacidad sensorial, con la finalidad de promover el fácil acceso a una ES. Sin embargo y a pesar de los grandes esfuerzos tanto de entes gubernamentales como instituciones privadas y sociedad en general el acceso a la ES de personas con discapacidad ha variado en torno a la baja, por ejemplo, en el año 2005 alcanzo el 1,8\%, en el 2009 llega al 1,9\% y en el 2015 baja al 1,2\% de estudiantes incluidos en ES (CONADIS, 2018).

Con todo lo expuesto se diría que el Ecuador cuenta con políticas y culturas inclusivas, pero es en la práctica donde el trabajo es más arduo y requiere del esfuerzo de toda la sociedad. Si bien en este trabajo se aborda la inclusión desde el enfoque de derecho para las personas con discapacidad, la palabra Inclusión en sí, refiere a todo alumno en general, considerando que todos y cada uno de los individuos son únicos y por lo tanto diferentes. Así, el modelo educativo que se pretende conseguir es aquel que no necesite de adaptaciones ni reformas, sino por el contrario sea plenamente claro, equitativo y permanente.

Un modelo de formación donde el estudiante con NEE asociadas o no a la discapacidad, no requiera una atención individualizada, sino sea dentro del mismo espacio y tiempo de clase de modo que el maestro planifique actividades con recursos y herramientas que permitan el desarrollo de capacidades y habilidades diversas, fruto de un grupo heterogéneo con inteligencias diversas. Esta transformación a la educación está provocando una innovación social que permitirá en algún momento y gracias al esfuerzo de todos que una -cultura educativa innovadora e inclusiva- sea una realidad nacional y una ley ejecutada y no simplemente plasmada en la Constitución.

La Universidad Católica de Cuenca [UCACUE], población con la que se trabajó para la realización de este artículo, cuenta con la Unidad de Diagnóstico, Investigación Psicopedagógica y Apoyo a la Inclusión [UDIPSAI] donde a través un equipo transdisciplinario de profesionales evalúan el caso, y continua con el proceso de inclusión, independientemente del origen de esa necesidad. Se Inicia con la adaptación 


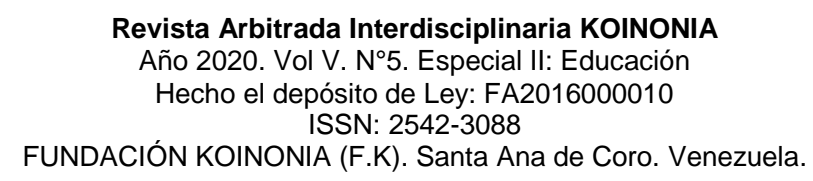

Catalina Patricia Muñoz-León; Darwin Gabriel García-Herrera; María Isabel Álvarez-Lozano Juan Carlos Erazo-Álvarez

Grado 1: Adaptación curricular de acceso mediante la información detallada en la ficha de Adaptación Curricular Individual en Educación Superior [ACIES], pasa al Grado 2: Adaptación en la planificación y actividades y si el caso lo amerita finaliza en un Grado 3: Adaptación en la evaluación e indicadores de logro. En este proceso intervienen todos los actores educativos: Autoridades, Administrativos, Docentes y Representantes. Es un departamento que trabaja juntamente con otras áreas como Bienestar Estudiantil encargado de velar por la titulación y adquisición de empleo del estudiante (García, 2017).

\section{METODOLOGÍA}

La presente investigación fue de tipo descriptiva no experimental de cohorte transversal ya que se realizó en un solo momento. La población estuvo conformada por 34 docentes de la Universidad Católica de Cuenca (UCACUE), aplicandose un muestreo aleatorio simple, como técnica de recolección de datos, se empleó la encuesta online y como instrumento, un cuestionario de 54 items y alternativas de respuesta en escala de Likert entre 1 (valor mínimo) a 5 (valor máximo).

Dicho instrumento fue validado en contenido por el juicio de expertos y en consistencia interna por el calculo de coeficiente de Alpha de Cronbach, obteniendose un resultado de 0.982, siendo considerado confiable para su aplicación. Adicionalmente se aplicó el analisis de normalidad de Shapiro-Wilk cuyos resultados fueron menores a 0.05 determinando que todas las variables son paramétricas. Los datos obtenidos de la aplicación del instrumento, fueron calculados a través de estadistica descriptiva y chi cuadrado de Pearson con apoyo del programa IBM SPSS Statistics V.20.

Luego de analizar los datos y determinar los resultados obtenidos con las respuestas más recurrentes se procedió a la realización de posibles propuestas estratégicas con la finalidad de aportar en el enriquecimiento de las políticas y prácticas inclusivas que la UCACUE ha ejecutado hasta el momento. 
Revista Arbitrada Interdisciplinaria KOINONIA

Año 2020. Vol V. N5. Especial II: Educación

Hecho el depósito de Ley: FA2016000010

ISSN: 2542-3088

FUNDACIÓN KOINONIA (F.K). Santa Ana de Coro. Venezuela.

Catalina Patricia Muñoz-León; Darwin Gabriel García-Herrera; María Isabel Álvarez-Lozano Juan Carlos Erazo-Álvarez

\section{RESULTADOS}

A partir de la encuesta realizada a docentes de la Unidad Academica [UA] de Educación de la UCACUE, se consideró el análisis de 8 variables relevantes, pues responden a la percepción de los docentes frente a la inclusión en la educación superior, objetivo de esta investigación. Cabe destacar que el formulario de preguntas responden a una adaptacion del "Index for Inclusion" originalmente en idioma ingles, misma que divide su analisis en tres dimensiones: Dimensión A:Culturas Inclusivas, Dimensión B: Políticas Inclusivas y Dimensión C: Prácticas Inclusivas.

\section{Tabla 1.}

Dimensión A: Crear Culturas Inclusivas

Relación entre: si la Carrera es accesible para todas las personas y si la Carrera implementa acciones para disminuir las prácticas discriminatorias.

\begin{tabular}{llrrrr}
\hline & & \multicolumn{2}{c}{ La Carrera implementa acciones para disminuir } & \\
& & \multicolumn{2}{c}{ las prácticas discriminatorias } & \\
\cline { 3 - 5 } & & Casi nunca & Regularmente & Frecuentemente & Total \\
\hline La Carrera es accesible & Frecuentemente & 0 & 1 & 19 & 20 \\
para todas las personas & Regularmente & 1 & 5 & 4 & 10 \\
& Casi nunca & 3 & 0 & 0 & 3 \\
& Nunca & 0 & 1 & 0 & 1 \\
\hline Total & & 4 & 7 & 23 & 34 \\
\hline
\end{tabular}

Pruebas de chi-cuadrado

\begin{tabular}{lccr}
\hline & Valor & gl & Sig. asintótica (bilateral) \\
\hline Chi-cuadrado de Pearson & $38,641^{\text {a }}$ & 6 & 0,000 \\
Razón de verosimilitudes & 30,419 & 6 & 0,000 \\
Asociación lineal por lineal & 18,327 & 1 & 0,000 \\
N de casos válidos & 34 & & \\
\hline a. 10 casillas (83,3\%) tienen una frecuencia esperada inferior a 5. La frecuencia mínima esperada es,12.
\end{tabular}

Fuente: Elaboración propia 
Revista Arbitrada Interdisciplinaria KOINONIA

Año 2020. Vol V. N5. Especial II: Educación

Hecho el depósito de Ley: FA2016000010

ISSN: 2542-3088

FUNDACIÓN KOINONIA (F.K). Santa Ana de Coro. Venezuela.

Catalina Patricia Muñoz-León; Darwin Gabriel García-Herrera; María Isabel Álvarez-Lozano Juan Carlos Erazo-Álvarez

Se observa que es una hipótesis afirmativa pues el valor de P es menor a 0.05 indicando una relación directa entre variables pues la carrera implementa acciones para disminuir las prácticas discriminatorias lo que provoca que sea un espacio de aprendizaje accesible a todas las personas. Sin embargo, las continuas investigaciones en torno al tema aportaran a mantener el espíritu inclusivo que promueve la institución en la actualidad.

\section{Tabla 2.}

Dimensión B: Elaborar Políticas Inclusivas

Relación entre: Si se implementan acciones para detectar las relaciones de abuso de poder o acoso escolar y si el profesorado recibe formación para gestionar la disciplina en el aula.

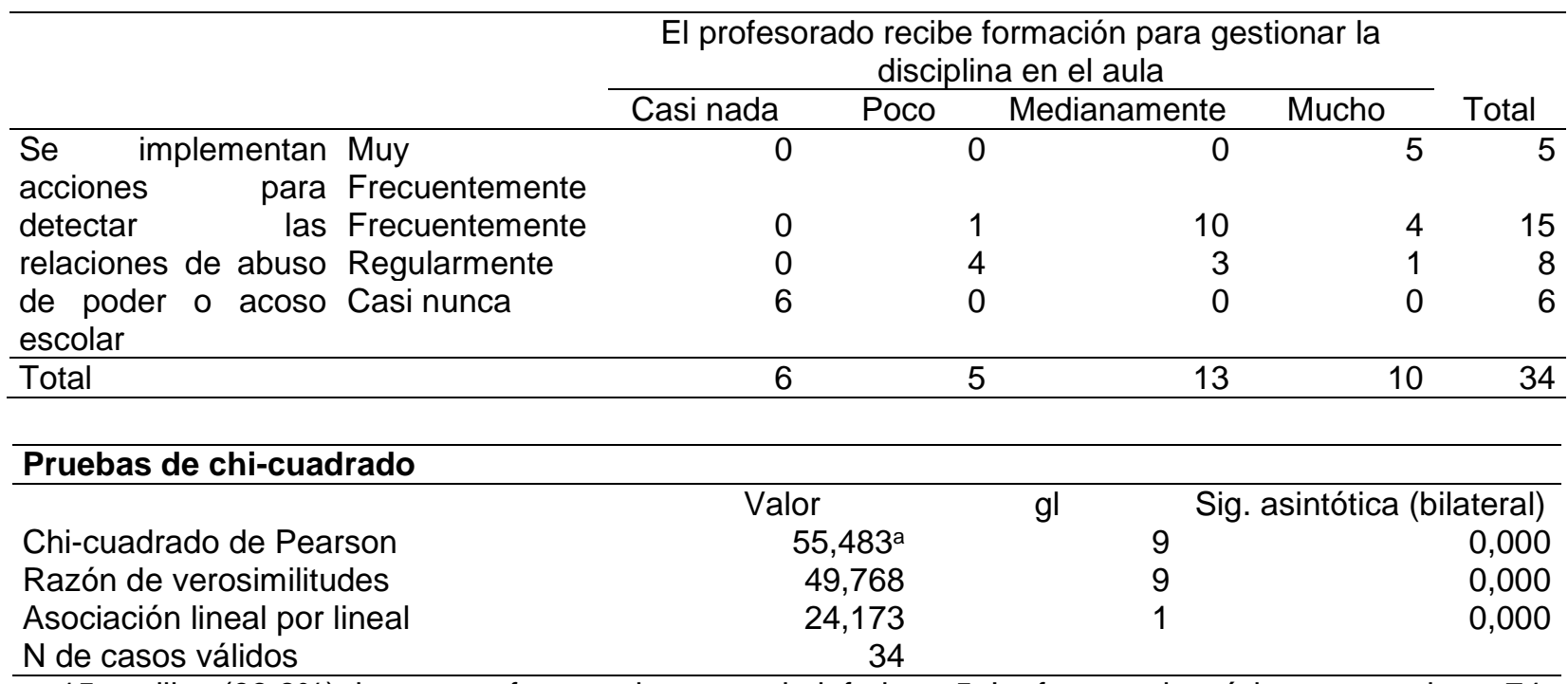

a. 15 casillas $(93,8 \%)$ tienen una frecuencia esperada inferior a 5. La frecuencia mínima esperada es,74.

Fuente: Elaboración propia.

Se observa que es una hipótesis afirmativa pues el valor de P es menor a 0.05 indicando una relación directa entre variables pues el profesorado recibe formación para gestionar la disciplina en el aula lo que provoca que se implementen acciones para detectar las relaciones de abuso de poder o acoso escolar. Es importante determinar los factores que provocan estas reacciones, así como las estrategias y acciones que se deben implementar para evitar riesgos y sanciones. 
Revista Arbitrada Interdisciplinaria KOINONIA

Año 2020. Vol V. N5. Especial II: Educación

Hecho el depósito de Ley: FA2016000010

ISSN: 2542-3088

FUNDACIÓN KOINONIA (F.K). Santa Ana de Coro. Venezuela.

Catalina Patricia Muñoz-León; Darwin Gabriel García-Herrera; María Isabel Álvarez-Lozano Juan Carlos Erazo-Álvarez

\section{Tabla 3.}

Dimensión C: Desarrollar Prácticas Inclusivas

Relación entre: Si los contenidos de las materias de estudio se adecuan a la diversidad del alumnado y si la diversidad del alumnado se utiliza como recurso para enriquecer el proceso educativo.

\begin{tabular}{|c|c|c|c|c|c|c|}
\hline & \multicolumn{5}{|c|}{$\begin{array}{c}\text { La diversidad del alumnado se utiliza como recurso para } \\
\text { enriquecer el proceso educativo }\end{array}$} & \multirow[b]{2}{*}{ Total } \\
\hline & Nada & $\begin{array}{l}\text { Casi } \\
\text { nada }\end{array}$ & Poco & Medianamente & Mucho & \\
\hline Los contenidos de Mucho & 0 & 0 & 0 & 0 & 10 & 10 \\
\hline las materias de Medianamente & 0 & 0 & 2 & 12 & 0 & 14 \\
\hline estudio $\quad$ se Poco & 0 & 0 & 5 & 1 & 0 & 6 \\
\hline 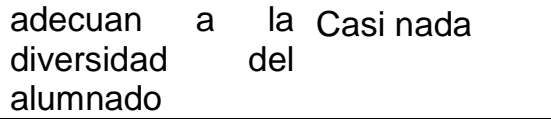 & 1 & 3 & 0 & 0 & 0 & 4 \\
\hline Total & 1 & 3 & 7 & 13 & 10 & 34 \\
\hline
\end{tabular}

\section{Pruebas de chi-cuadrado}

Chi-cuadrado de Pearson

Valor

$82,963^{a}$

71,829

29,548

Asociación lineal por lineal

$\mathrm{N}$ de casos válidos

34

$\begin{array}{rrr}\text { gl } & \text { Sig. asintótica (bilateral) } \\ 12 & 0,000 \\ 12 & 0,000 \\ 1 & 0,000 \\ & \end{array}$

a. 19 casillas (95,0\%) tienen una frecuencia esperada inferior a 5. La frecuencia mínima esperada es 12.

Fuente: Elaboración propia.

Se observa que es una hipótesis afirmativa pues el valor de $\mathrm{P}$ es menor a 0.05 indicando una relación directa entre variables pues la diversidad estudiantil es considerada una riqueza dentro del proceso educativo lo que provoca que los contenidos de las materias de estudio se adecúen a las necesidades y habilidades del estudiante. Para complementar este proceso se sugiere evaluar periódicamente la actualización de dichos contenidos y mantener la calidad educativa. 


\section{Catalina Patricia Muñoz-León; Darwin Gabriel García-Herrera; María Isabel Álvarez-Lozano Juan Carlos Erazo-Álvarez}

\section{Tabla 4.}

Dimensión A: Crear Culturas Inclusivas

Toda la comunidad universitaria se identifica con la filosofía, los principios y los objetivos de la educación inclusiva.

\begin{tabular}{llrrrr}
\hline & & & & Porcentaje \\
Válidos & Frecuencia & Porcentaje & Porcentaje válido & acumulado \\
\cline { 3 - 6 } & Medianamente & 8 & 23,5 & 23,5 & 23,5 \\
& 16 & 47,1 & 47,1 & 70,6 \\
& Poco & 5 & 14,7 & 14,7 & 85,3 \\
& Casi nada & 5 & 14,7 & 14,7 & 100,0 \\
\cline { 2 - 6 } & Total & 34 & 100,0 & 100,0 & \\
\hline
\end{tabular}

Fuente: Elaboración propia.

Se observa que el personal encuestado medianamente se identifica con la filosofía de inclusión que mantiene la universidad alcanzando un 47,1\%. Aunque es un índice alto esto podría superarse mediante la divulgación continua de las políticas inclusivas que establece la institución. El involucramiento de toda la comunidad universitaria es fundamental.

\section{Tabla 5.}

Dimensión B: Elaborar Políticas Inclusivas

Se promueve la investigación y formación del profesorado en temas relacionados con la educación inclusiva.

\begin{tabular}{llrrrr}
\hline & & & & Porcentaje \\
Válidos & Frecuencia & Porcentaje & Porcentaje válido & acumulado \\
\cline { 3 - 6 } & Medianamente & 10 & 29,4 & 29,4 & 29,4 \\
& Poco & 12 & 35,3 & 35,3 & 64,7 \\
& Casi nada & 7 & 20,6 & 20,6 & 85,3 \\
& Total & 5 & 14,7 & 14,7 & 100,0 \\
\hline
\end{tabular}

Fuente: Elaboración propia. 


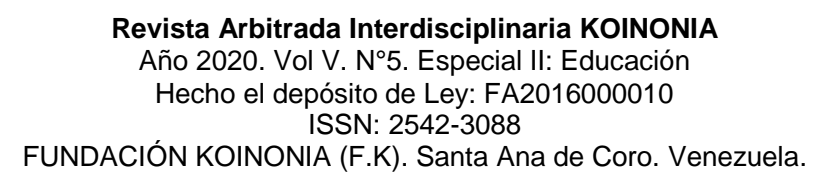

Catalina Patricia Muñoz-León; Darwin Gabriel García-Herrera; María Isabel Álvarez-Lozano Juan Carlos Erazo-Álvarez

Se observa que la investigación y formación docente en torno a la educación inclusiva alcanza el índice más alto (35,3\%) sin embargo se encuentra dentro del punto sobre la media, que resalta la necesidad de fomentar en los docentes esa cultura investigativa que permita empoderarse de esa herramienta clave del conocimiento y poder acoger debidamente la discapacidad en sus aulas.

Se diría que la inclusión en el campo universitario toma fuerza cada vez mayor debido a reglamentos internacionales y locales que poco a poco han ido socializando el nuevo enfoque educativo en el quehacer docente, administrativo y sociedad en general. Los resultados obtenidos en esta investigación evidencian índices superiores a la media en torno a la inclusión dentro del campus universitario. Esto ocurre gracias a la existencia del departamento UDIPSAI que incita a mirar a todo integrante como un individuo con habilidades nuevas, diferentes y propias, sin permitir que una discapacidad sea lo que define al estudiante.

Cabe destacar que los encuestados fueron entre docentes, personal administrativo y autoridades de la Unidad Académica [UA] de Educación en la matriz de la ciudad de Cuenca, la sede en Azogues y las extensiones en Cañar y Macas. Se evidencia que la percepción de los docentes en torno a la EIS es relativamente alta, lo cual indica que la UA posee una cultura inclusiva gracias a sus políticas y prácticas a favor de la inclusión. Todo esto indica un trabajo interno importante que podría compararse con lo realizado por (Leguizamón, et al., 2019), la Universidad de la Plata [UNPL] donde luego de realizar encuestas a sus docentes sobre sus percepciones y experiencias frente a un alumno con discapacidad fueron del mismo modo favorables gracias a la capacitación, difusión y asesoramiento en torno a la discapacidad que realiza la Facultad de Humanidades y Ciencias de la Educación a través de su Comisión Universitaria de Discapacidad [CUD]. Se evidencia que, contar con un espacio que brinde asesoría y seguimiento en torno a la educación inclusiva, desemboca en una doble confianza, el acceso y formación sin restricciones para todo estudiante y una práctica docente de calidad sin limitaciones para todo maestro. 


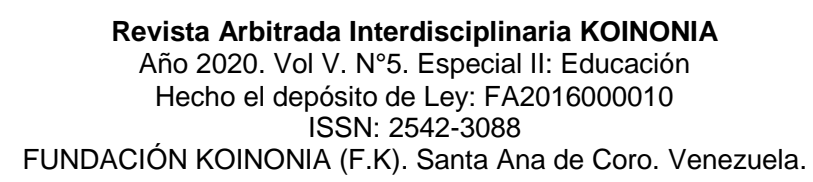

Catalina Patricia Muñoz-León; Darwin Gabriel García-Herrera; María Isabel Álvarez-Lozano Juan Carlos Erazo-Álvarez

\section{PROPUESTA}

La inclusión en la educación no deja de ser un reto que requiere arduo trabajo y participación. Sin importar la velocidad con la que se implemente una educación inclusiva, es importante la toma de conciencia, iniciativa y voluntad. Incluir a una persona con discapacidad dentro del sistema educativo exige responsabilidad en asegurar un aprendizaje de calidad, pero de manera compartida con el resto de los alumnos, asegurando la eliminación de todo tipo de barrera ya sea de: acceso, recursos, organización y acción.

Dentro de las instituciones de educación superior de la región del austro, la UCACUE es una de las más extensas, lo que invita a tomar ventaja sobre esta característica pues los estudios que en ella se realicen ofrecerán resultados desde la perspectiva de un número importante de profesores que ejercen su labor en varios contextos de la región.

A partir de los resultados obtenidos de la encuesta donde en su mayoría participaron docentes de las carreras de Psicología Educativa 26,5\%; P.A. Física y el Deporte 23,5\%; y Educación 20,6, se evidencia que en la institución se cumple con la Dimensión A: Creación de culturas inclusivas, pues casi la totalidad de los encuestados respondieron sentirse acogido dentro del establecimiento, de igual manera en cuanto a la Dimensión B: Elaborar políticas inclusivas, la respuesta fue positiva ya que los maestros se sienten identificados con la filosofía inclusiva a través de las distintas actividades y gestiones en favor de los alumnos de inclusión.

Se cree pertinente trabajar en la Dimensión C: Desarrollo de prácticas inclusivas, ya que está directamente ligada al proceso enseñanza aprendizaje. Hay que reconocer entonces los principales actores de este proceso, para ello la siguiente propuesta se divide en dos grupos: maestros y estudiantes. Todo esto se gestionará a través del departamento UDIPSAI a manera de gestiones paralelas que complementan lo realizado por la UCACUE hasta el momento. 
Catalina Patricia Muñoz-León; Darwin Gabriel García-Herrera; María Isabel Álvarez-Lozano Juan Carlos Erazo-Álvarez

1) Maestros: Se propone la creación de una red interna de maestros bajo el nombre “Progreso Docente" bajo el eslogan, "La práctica inclusiva, no es dar ayuda o afecto, es dar dignidad y respeto.". Una gestión de inclusión activa que convoque a la articulación de maestros en favor de la inclusión en las aulas en respuesta al nuevo modelo educativo para todos. Dentro del programa los profesores deberán cumplir los siguientes pasos:
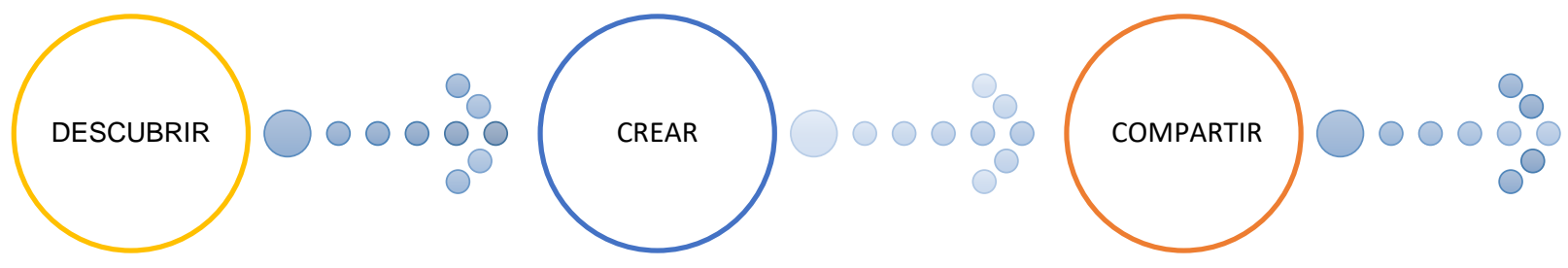

Figura 1. Proceso para mejorar la practica inclusiva desde los docentes. Fuente: Elaboración propia.

1. DESCUBRIR. - investigar y conocer sobre la discapacidad de su alumno, pero también aquellas cualidades, aptitudes y fortalezas que le permitirán su acceso a al ámbito educativo superior y lograr una inserción laboral.

2. CREAR. - ambientes, actividades y estrategias que permitan empatizar con el alumno de inclusión. Comenzar principalmente con el uso de una correcta terminología, partiendo claro esta del entender que todo ser humano tiene su propia palabra de identificación que es básicamente su nombre, de tal modo que no sea la discapacidad lo que define al estudiante sino su identidad personal y calidad humana.

3. COMPARTIR. - aportar con sus experiencias a transformar la expectativa de vida de los alumnos incluidos, pero también a una mejor percepción de los docentes 
Catalina Patricia Muñoz-León; Darwin Gabriel García-Herrera; María Isabel Álvarez-Lozano Juan Carlos Erazo-Álvarez

frente a la discapacidad en la universidad. Un trabajo Colaborativo que permita crear esa red interna de profesores empoderados en conocimientos académicos, pero también en habilidades socioemocionales como empatía, colaboración y toma de decisiones en busca de la autonomía del alumno.

\section{Se recomienda:}

a) Que al final de cada ciclo el maestro realice un escrito o relato de su experiencia, con las dificultades y retos que surgieron al momento de tener un estudiante de inclusión en su aula.

b) Que al final en un escrito o relato el docente comparta los logros alcanzados por el alumno/a incluido/a y como esta vivencia, enriqueció su labor docente y su percepción de la discapacidad.

c) Que la participación en este programa sea de libre acceso y voluntario

d) Que al participar en este programa el docente reciba un reconocimiento a manera de certificación interna que indique ser un Docente Inclusivo,

e) Que el mencionado certificado tenga un reconocimiento interno de valor para su currículo, y sirva de ejemplo a seguir del resto de colegas docentes.

2) Estudiantes: de igual manera se propone la creación de un programa para estudiantes. Un grupo de diálogo y encuentro juvenil bajo el nombre "Progreso Estudiantil" con el eslogan, "Todos para uno y uno para todos.". Una participación en apoyo a la inclusión desde los jóvenes y para los jóvenes. Se trata de un trabajo para animar a la comunidad estudiantil a aprender a convivir con la discapacidad desde la tolerancia, aceptación y acción. Se trata de jóvenes capacitando a sus pares en torno a la discapacidad y cualquier otro tipo de diferencias que se presentan en los grupos humanos. Reconocer las dificultades de las personas con alguna necesidad física, emocional o intelectual, pero de igual manera poner atención a las habilidades que son precisamente lo que le hace especial y único en la sociedad.

Dentro del programa los estudiantes deberán escalar los siguientes pasos: 


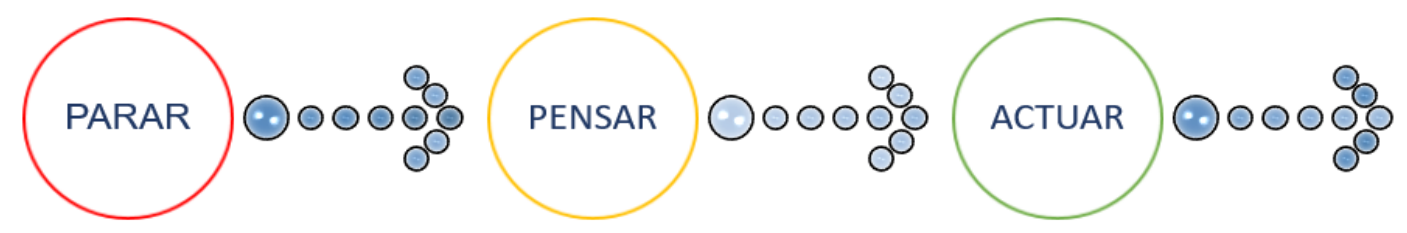

Figura 2. Procesos para mejorar la practica inclusiva desde los estudiantes.

Fuente: Elaboración propia.

a) PARAR. - para reflexionar y hacer un alto en sus actitudes y actividades. Deberán indagar a través de encuentros diálogos, observación, encuestas y otros las percepciones de los estudiantes ante la diversidad. conocer el nivel de tolerancia frente a la discapacidad y el nivel de resiliencia de las personas que la enfrentan, esto para mejorar o cambiar la manera de interactuar con y entre los alumnos que presentan alguna situación de vulnerabilidad.

b) PENSAR. - para reaccionar y proponer posibles soluciones comportamentales y actitudinales ante situaciones de rechazo o acoso que podrá darse en la universidad. Planificar encuentros de dialogo estudiantiles para evolucionar en las prácticas de convivencia dentro del establecimiento educativo. Creación de talleres de capacitación a los estudiantes de las distintas carreras y momentos de participación en actividades que requieran mayor atención a personas con discapacidad.

c) ACTUAR. - para redireccionar y reestructurar juntamente con los distintos actores educativos (Docentes, profesionales expertos, personal administrativo, autoridades y familias) la participación de todos los estudiantes de inclusión a manera de testimonios de vida como han tenido que enfrentar las acciones frente a su condición, dentro y fuera de la institución. estos encuentros, así como mantenerse vigilantes del cumplimiento de las normativas que amparan a las personas con discapacidad. El apoyo y/o acompañamientos a los alumnos con 


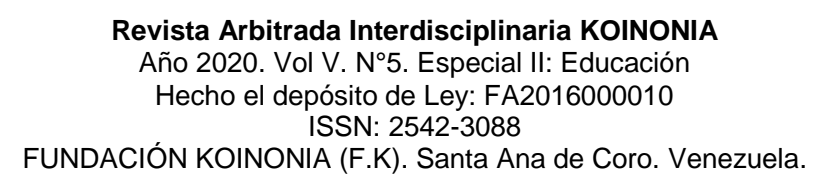

Catalina Patricia Muñoz-León; Darwin Gabriel García-Herrera; María Isabel Álvarez-Lozano Juan Carlos Erazo-Álvarez

discapacidad con la designación de un alumno-colaborador/a que sea quien asesore al alumno incluido en las distintas tareas, programas, y gestiones universitarias que se realice. Los jóvenes podrán acceder y formar parte activa de un cambio local de vida y no simplemente, conocer de manera general sobre inclusión y discapacidad.

Se recomienda:

a) Que el ingreso a este club sea voluntario y de libre acceso y participación.

b) Que el propósito del club se centre en la realización de actividades y estrategias que difundan la importancia de una práctica inclusiva en todos los espacios y momentos desde el compañerismo y solidaridad.

c) Que la participación en este club sirva de tiempo parcial de las horas de prácticas preprofesionales que exige la malla curricular.

d) Que los estudiantes que participen en este club reciban un reconocimiento como certificado que avale su preparación en términos inclusivos y se convierta en un documento de importancia dentro de su currículo.

\section{CONCLUSIONES}

Se determina que los docentes de la Unidad Académica de Educación de la Universidad Católica de Cuenca [UCACUE], poseen percepciones positivas frente a la inclusión debido a la cantidad de alumnado con varias discapacidades que acoge la universidad, en su totalidad 154. Adicionalmente estos resultados se logran gracias a la difusión de políticas inclusivas internas y el trabajo conjunto que realizan los departamentos de Bienestar Estudiantil y la Unidad de Diagnóstico, Investigación Psicopedagógica y Apoyo a la Inclusión [UDIPSAI] aportando de manera directa a la disminución de barreras y retos que enfrentan los docentes dentro de sus aulas.

Lo anterior permite afirmar la hipótesis planteada pues los docentes están conscientes de la importancia de aplicar con éxito el nuevo modelo educativo basado en la inclusión de todas y todos los estudiantes sin ningún tipo de exclusión o segregación. 


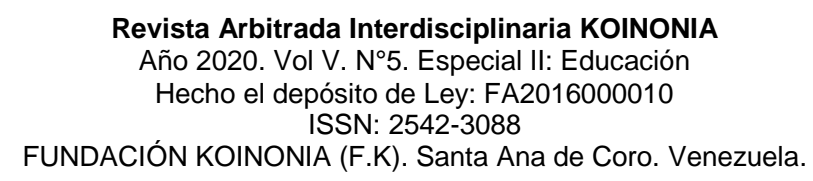

Catalina Patricia Muñoz-León; Darwin Gabriel García-Herrera; María Isabel Álvarez-Lozano Juan Carlos Erazo-Álvarez

No obstante, esto no obvia el hecho de que las prácticas inclusivas deben expandirse desde los docentes y autoridades hacia los estudiantes y resto de comunidad universitaria. A fin de evitar que en un futuro se convierta en un inconveniente en la convivencia académica y clima estudiantil.

En este sentido el estudio de la percepción laboral de los docentes frente a la discapacidad permite el siguiente diagnóstico.

En lo que se refiera a la Dimensión A: crear culturas inclusivas la UA de Educación implementa acciones para prevenir riesgos psicosociales y disminuir las prácticas discriminatorias, aportando al control y cuidado de un ambiente cordial, respetuoso y solidario. Las expectativas hacia el alumnado son altas instando a los docentes a implementar acciones para eliminar las barreras al aprendizaje y la participación. Todo esto de manera conjunta con UDIPSAI y los protocolos y sugerencias que determine. Con respecto a la Dimensión B: elaboración de políticas inclusivas, se constata que la UA programa de manera periódica, actividades abiertas a la comunidad a manera de difundir su trabajo y aportar a la población del entorno. Además, una vez que el estudiante ingresa es debidamente atendido y direccionado en todas sus actividades de manera que se garantice su preparación y formación tanto laboral como humana. Los recursos y servicios que ofrece la UA se ajustan a las necesidades de los estudiantes y una realidad social cambiante. Por otro lado, los docentes acuerdan que la institución permite su escala profesional con nombramientos y promociones de mayor nivel y responsabilidad. El apoyo psicopedagógico al estudiante se lo realiza de manera frecuente según sus requerimientos al igual que al docente y la familia en caso de requerirlo. Esto hace que en ocasiones los procedimientos tradicionales de evaluación se combinen con otros o que la asignatura pueda ser cursada de diferente manera a la presencial, como lo es mediante tutorías, una vez claro está, se solicite y, se entregue la documentación necesaria de soporte.

En cuanto a la Dimensión C: desarrollo de prácticas inclusivas se determina que frecuentemente los contenidos de las materias están relacionados a problemas sociales 
relevantes y se adecuan al estudiante o grupos de aprendizaje diversos. Del mismo modo se organiza espacios y tiempos a favor de la comunidad estudiantil para instar a formar parte activa de estos momentos. La diversidad del alumnado es utilizada como una ventaja a favor del enriquecimiento de la experiencia docente y el proceso educativo que brinda la UCACUE.

\section{FINANCIAMIENTO}

No monetario

\section{AGRADECIMIENTO}

A la Universidad Católica de Cuenca; por apoyar el desarrollo de la investigación.

\section{REFERENCIAS CONSULTADAS}

Asamblea Nacional. (2008). Constitución de la República del Ecuador 2008. [Constitution of the Republic of Ecuador 2008]. Recuperado de https://n9.cl/hd0q

Booth, T., \& Ainscow , M. (2002). Index for Inclusion: developing learning and participation in schools. [Indice de inclusión: desarrollo del aprendizaje y la participación en las escuelas]. CSIE, 1-106. Recuperado de https://n9.cl/lo1en

Clavijo-Castillo, R. G., \& Bautista-Cerro, M. J. (2020). La educación inclusiva. Análisis y reflexiones en la educación superior ecuatoriana. [Inclusive education. Analysis and reflections on Ecuadorian higher education]. ALTERIDAD. Revista de Educación, 15(1), 113-124. https://doi.org/10.17163/alt.v15n1.2020.09

CONADIS. (2018). Informe de Rendición de Cuentas 2018. [Accountability report 2018]. Recuperado de https://n9.cl/42g8o

Cotán, A. (2017). Educación inclusiva en las instituciones de educación superior:narrativas de estudiantes con discapacidad. [Inclusive education in higher education institutions: narratives of students with disabilities]. Revista Española de Discapacidad (REDIS), 5(1), 43-61. https://doi.org/10.5569/2340-5104.05.01.03 
Revista Arbitrada Interdisciplinaria KOINONIA

Año 2020. Vol V. N5. Especial II: Educación

Hecho el depósito de Ley: FA2016000010

ISSN: 2542-3088

FUNDACIÓN KOINONIA (F.K). Santa Ana de Coro. Venezuela.

Catalina Patricia Muñoz-León; Darwin Gabriel García-Herrera; María Isabel Álvarez-Lozano Juan Carlos Erazo-Álvarez

CRES. (2018). Conferencia Regional de Educación Superior de América Latina y el Caribe- Córdoba, 2018 (Resúmenes Ejecutivos). [Regional Conference on Higher Education of Latin America and the Caribbean- Córdoba, 2018 (Executive Summaries)]. Recuperado https://n9.cl/douew

Echeita, G., \& Ainscow, M. (2011). La educación inclusiva como derecho: Marco de referencia y pautas de acción para el desarrollo de una revolución pendiente. [Inclusive education as a right.: Framework and guidelines for action for the development of a pending revolution]. Tejuelo: Didáctica de la Lengua y la Literatura. Educación, (12), 26-46.

García, W. (2017). Adaptaciones curriculares en las aulas de educación superior como base para la inclusión de estudiantes con discapacidad. [Curricular adaptations in higher education classrooms as a basis for the inclusion of students with disabilities]. Universidad Católica de Santiago de Guayaquil. Recuperado de https://n9.cl/z7mr

Gross-Martínez, M., \& Stiller-González, L. (2014). Experiencia de Trabajo Colaborativo: Formación del Personal Docente Universitariopara la Diversidad. [Collaborative Work Experience: Training of University Teaching Staff for Diversity]. Revista de Ciencias Sociales, (143), 31-41. https://doi.org/10.15517/rcs.v0i143.14568

Leguizamón, M., Katz, S., Giglio, M., Arouxet, B., Innaro, M., Musicco, D., González, E. (2019). Los docentes y la accesibilidad de los estudiantes en situación de discapacidad en la UNLP. [Teachers and the accessibility of students with disabilities at UNLP]. 13 Congreso Argentino de Educación Física y Ciencias, 30 de septiembre al 4 de Octubre de 2019. Universidad Nacional de la Plata. Facultad de Humanidades y Ciencias de la Educación. Departamento de Educación Física, Ensenada, Argentina, Argentina. Recuperado de https://n9.cl/13kv

LOES. (2010). Ley Organica De Educacion Superior, LOES. [Organic Law Of Higher Education, OLHE]. Registro Oficial Suplemento 298 de 12-oct.-2010. Recuperado de https://n9.cl/gotv

MINEDUC. (2011). Módulo I: educación inclusiva y especial. [Module I: inclusive and special education]. Recuperado de https://n9.cl/koxcy

Moriña, A., \& Melero, N. (2016). Redes de apoyo sociales y académicas de estudiantes con discapacidad que contribuyen a su inclusión en la enseñanza superior. [Social and academic support networks of students with disabilities that contribute to their inclusion in higher education]. Revista Prisma Social, (16), 32-59. 
Revista Arbitrada Interdisciplinaria KOINONIA

Año 2020. Vol V. N5. Especial II: Educación

Hecho el depósito de Ley: FA2016000010

ISSN: 2542-3088

FUNDACIÓN KOINONIA (F.K). Santa Ana de Coro. Venezuela.

Catalina Patricia Muñoz-León; Darwin Gabriel García-Herrera; María Isabel Álvarez-Lozano Juan Carlos Erazo-Álvarez

Ocampo, J. (2018). Discapacidad, Inclusión y Educación Superior en Ecuador: El Caso de la Universidad Católica de Santiago de Guayaquil. [Disability, Inclusion and Higher Education in Ecuador: The Case of the Catholic University of Santiago de Guayaquil]. Revista latinoamericana de educación inclusiva, 12(2), 97-114. https://dx.doi.org/10.4067/S0718-73782018000200097

OMS. (2011). Informe mundial sobre la discapacidad. [World report on disability]. Recuperado https://n9.cl//r54

Organización de las Naciones Unidas para la Educación, la Ciencia y la Cultura, UNESCO. (2015). Educación 2030. Declaración de Incheon. Hacia una educación inclusiva y equitativa de calidad y un aprendizaje a lo largo de la vida para todos. [Education 2030. Incheon Declaration. Towards inclusive and equitable quality education and lifelong learning for all]. Recuperado de https://n9.cl/fbqik

Organización de las Naciones Unidas para la Educación, la Ciencia y la Cultura, UNESCO. (2019). Inclusión en la educacion. [Inclusion in education]. https://es.unesco.org/themes/inclusion-educacion

Organización de las Naciones Unidas. (2008). La educación inclusiva: el camino hacia el futuro. [Inclusive education: the way to the future]. Recuperado de https://n9.cl/3syt

Rafy-Syed-Jaafar, S.M., Maarof, R, Juzaili-Hisam, M, Annysa-Husin, N. (2020). Inclusion Setting for Disabilities in Higher Education: A Systematic Review of Issues and Challenges in Disabilities Research. Advances in Social Science, Education and Humanities Research, 470, 1-7. https://dx.doi.org/10.2991/assehr.k.200921.030

Rojas-Rojas, L., Arboleda-Toro, N., \& Pinzón-Jaime, L. (2018). Characterization of a Population with Visual, Auditory, Speech and Motor Disabilities, for Their Linking to Undergraduate Programs at a University in Colombia. Revista Electrónica Educare, 22(1), 1-28. https://doi.org/10.15359/ree.22-1.6

Secretaría Nacional de Planificación y Desarrollo - Senplades (2017). Plan Nacional de Desarrollo 2017 - 2021 Toda una Vida [National Development Plan 2017 - 2021 A Lifetime]. Recuperado de https://n9.cl/znx4

Svendby, R. (2020). Lecturers' Teaching Experiences with Invisibly Disabled Students in Higher Education: Connecting and Aiming at Inclusion. Scandinavian Journal of Disability Research, 22(1); 275-284. http://doi.org/10.16993/sjdr.712 
Revista Arbitrada Interdisciplinaria KOINONIA

Año 2020. Vol V. N5. Especial II: Educación

Hecho el depósito de Ley: FA2016000010

ISSN: 2542-3088

FUNDACIÓN KOINONIA (F.K). Santa Ana de Coro. Venezuela.

Catalina Patricia Muñoz-León; Darwin Gabriel García-Herrera; María Isabel Álvarez-Lozano Juan Carlos Erazo-Álvarez

UIS. (2020). Data for the Sustainable Development Goals. [Datos para los Objetivos de Desarrollo Sostenible]. Recuperado de https://n9.cl/yuc6i

UNESCO-IESALC. (2018). Informe de Gestion 2011-2018 [Management Report 20112018]. https://n9.cl//9dy

Vélez-Calvo, X., Tárraga-Mínguez, R., Fernández-Andrés, M. I., \& Sanz-Cervera, P. (2016). Formación inicial de maestros en Educación Inclusiva: Una comparación entre Ecuador y España. [Initial Teacher Training in Inclusive Education:A Comparison Between Ecuador and Spain]. Revista de Educación Inclusiva, 9(3), 75-94.

Vilchis-Romero, V., \& Arriaga-Ornelas, J. (2018). Vivir y enfrentar la integración/exclusión educativa en el nivel medio superior: estudios de caso en jóvenes de San Luis Potosí, México. [Living and facing educational integration / exclusion at the upper secondary level: case studies in young people from San Luis Potosí, Mexico]. Revista Educación, 42(1),1-28.

(C2020 por los autores. Este artículo es de acceso abierto y distribuido según los términos y condiciones de la licencia Creative Commons Atribución-NoComercial-Compartirlgual 4.0 Internacional (CC BY-NC-SA 4.0) (https://creativecommons.org/licenses/by-nc-sa/4.0/). 